\title{
Modeling and Analysis of A Multi-Level Caching in Distributed Database Systems
}

\author{
Doaa S. El Zanfaly \\ Info. Systems Dept. \\ Faculty of Computers \& Info. \\ Helwan Univ., Cairo
}

\author{
Reda A. Ammar \\ Computer Science. \& Engr. Dept. \\ Univ. of Connecticut, Storrs, CT \\ 06269-1155
}

\author{
A. Sharaf Eldin \\ Info. Systems Dept. \\ Faculty of Computers \& Info. \\ Helwan Univ., Cairo
}

\begin{abstract}
Caching frequently asked queries is an effective way to improve the performance of both centralized and distributed database systems. Intensive works have been done in this area to propose different query caching techniques and to evaluate their performance. However, most of these works were confined to caching previous query results in a single-level caching architecture. Evaluations of these works were based on simulations. In [1], we proposed a new query caching technique for caching both query results and execution plans in a multi-level caching architecture. The centralized version of this technique was evaluated and the results were reported in [2]. In this paper, we present an analytical model to evaluate the performance of the proposed technique in distributed database systems.
\end{abstract}

Key-Words: - Multi-Level Caching, Query Caching, Query Processing, Performance Modeling.

\section{Introduction}

Most query caching techniques are confined to caching prior query results in a single level caching architecture to avoid accessing the underlying database each time a user submits the same query [3-7]. Although these techniques play a vital role in improving the performance of distributed queries, they do have some drawbacks. Caching query results needs space and an appropriate updating strategy to always keep cached results consistent with the underlying databases [4]. To overcome these drawbacks, we developed a new query caching approach that is based on caching query execution plans and some results in a multi-level caching architecture [1]. We also developed an analytical model to evaluate the performance of the new architecture when it is implemented to run on a single computer [2]. In this paper, we describe a new multi-level caching technique. Then, we present the analytical model to evaluate the performance of this technique when it is implemented to run in distributed systems. The results show that the multi-level caching technique achieves high performance outcomes.

\section{Multi-Level Caching Technique}

Instead of caching prior query results in a single-level cache architecture, we developed a new caching technique that caches query execution plans, subplans, and results in a multi-level cache architecture. By dividing the cache linearly into multiple levels, each level contains a subset of global queries subplans. Plans are cached in the form of interrelated but independent subplans. Subplans are interrelated as they all together represent a global execution plan. At the same time, they are independent as each subplan can be reused by itself to construct another execution plan with some other cached subplans. Each cache level will be further divided into two partitions: one for caching query execution plans (subplans) and the other for caching the results of those plans (subplans) that are frequently used. New plans are cached on the top cache level. As the system is being used, subplans will be distributed among different cache levels according to their recency. Through this architecture, we extend the idea of query caching by doing the following:

- We cache a combination of query results and execution plans. Caching query results reduces the access time. Caching execution plans makes the cache independent of the database. Each time data in a database is modified, there is no need to propagate these modifications to the cache. The plan will be reused whenever needed with its new attributes.

- We cache global plans and hence some of its subplans will be implicitly cached. Thus, cached plans can be reused to execute different queries instead of being used to execute only a sole general query. This will reduce the processing time by avoiding optimization and reconstruction of global execution plans (subplans). It also reduces the search time since we look into some parts of the cache instead of searching all of it. 


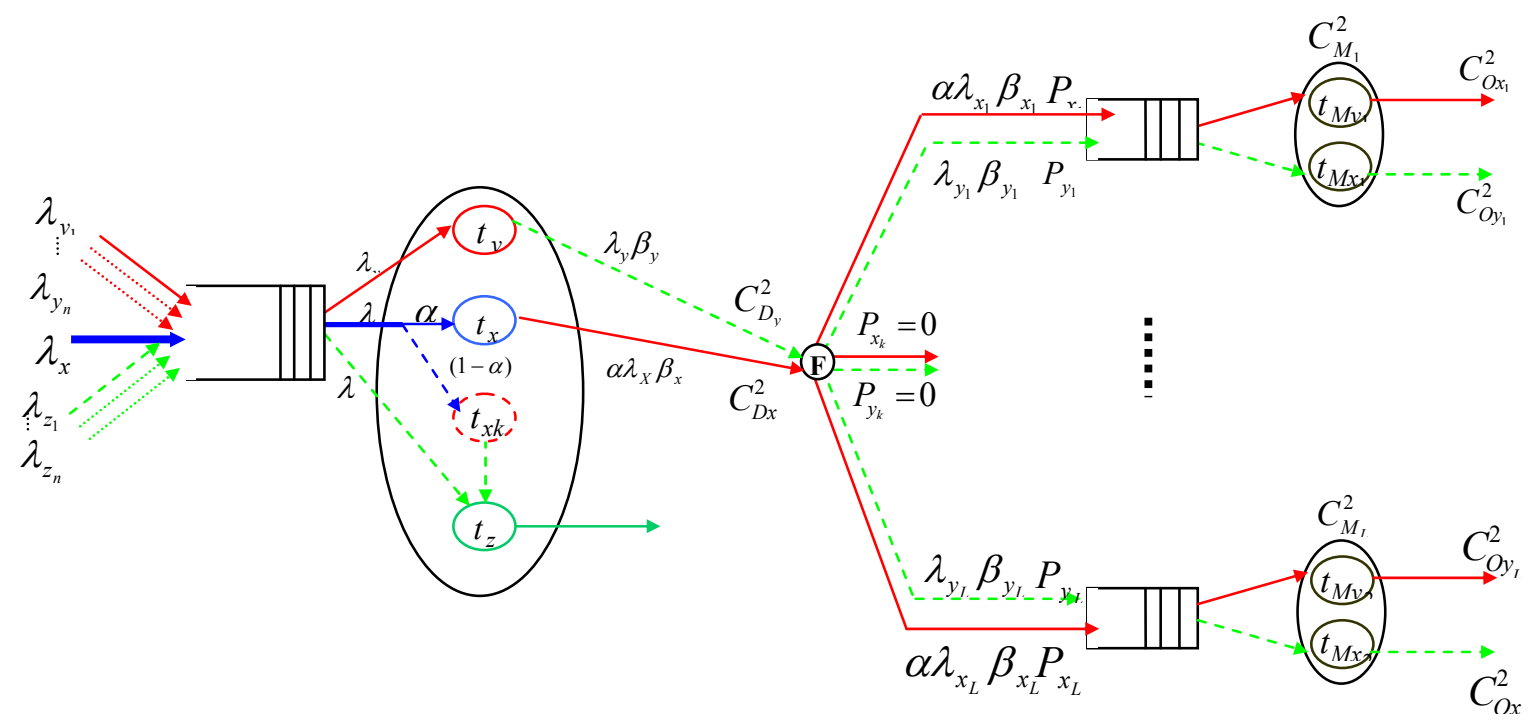

Figure 1: The Distributed Database Model

\section{Related works on Performance Modeling and Analysis}

As stated in a survey made by Nicola and Jarke [8], different analytical performance models, as well as simulations models, have been developed to estimate the performance of distributed database systems for different applications. These studies commonly use queuing systems as the underlying models. In [9] [10], a fully replicated database of $\mathrm{m}$ local sites was modeled by a $\mathrm{M} / \mathrm{M} / \mathrm{m} / \mathrm{FCFS}$ queuing system. Where the arrived queries follow Poisson distribution, served on a first-come-first-serve basis by $m$ servers and require an exponentially distributed service time. In these models, all sites share a single queue of incoming queries and the intersite communication is neglected. While others modeled distributed databases using open queuing networks in [11]. Some studies used networks of $\mathrm{M} / \mathrm{M} / \mathrm{I}$ queues so that each local database is modeled as an $\mathrm{M} / \mathrm{M} / 1$ system [12] [13] [14]. However, this restricts all requests to having the same exponentially distributed service time. More generally, some studies modeled the local databases as $\mathrm{M} / \mathrm{G} / 1 / \mathrm{FCFS}$ [15] and M/G/1/RR [16] queues with generally distributed service time. However, most of these models are based on the homogeneity assumption where all database sites and their respective workload are identical [8]. This assumption is not made in our model. Our model is a general model that can be applied to different distributed database architectures.

\section{Performance Model}

Most simulation and analytical performance studies of distributed database systems were based on the homogeneity assumption where all database sites and their respective workloads are identical. As stated in [14], this assumption implies the following: All database sites have the same structure, the same service capacity and hold the same amount of data. All sites receive the same workload, i.e. have an identical transaction arrival rate. Communication between the database sites is symmetrical. Unlike these models, our model doesn't follow the homogeneity assumption. It can be applied in both homogeneous and heterogeneous distributed system.

We modeled the distributed database system using an open network of queues. Each queue is represented by an M/G/1 FCFS mode. This is due to two reasons:

- Poisson streams have been found to be a good approximation for the arrival of queries submitted to the system due to a large number of users [8].

- The time required to process a request at a database site follows the general distribution, as it is non-deterministic.

- Considering a database system distributed among $n$ nodes, each node is represented as a two-stage tandem queues as in Figure 1. Each queue is a firstcome first-serve, single server queue. The first queue is the execution queue that can be modeled as an $\mathrm{M} / \mathrm{G} / 1$ queue. The second queue is the communication queue that can be modeled as a $\mathrm{G} / \mathrm{G} / 1$ queue. In the following two subsections we evaluate the average response time of each queue.

\subsection{Execution Queue}

As shown in Figure 2, three different classes enter the execution queue:

- The first class, class $x$, represents a global query received from a user with arrival rate $\lambda_{x}$. Portion of this class represents subqueries that will be processed at other nodes with probability $\alpha$. Other portion 
represents the share of node $k$ from the global query that will be processed locally with probability $(1-\alpha)$.

- The second class, class $y$, represents subqueries received from other nodes to be evaluated at node $k$. The arrival rate of this class is $\lambda_{y_{i}}$, where $i$ is a database nodes other than node $k$.

- The third class, class $z$, represents responses from other nodes to subqueries sent by node $k$. The arrival rate of this class is $\lambda_{z_{i}}$.

Hence, processing at the execution server can be decomposed into four main processes:

- Processing global queries, received from a user, with expected execution time $t_{x}$ and probability $\alpha$.

- Processing subqueries received form other nodes with expected execution time $t_{y}$.

- $(1-\alpha)$ is the probability that node $k$ is involved in the global query; the site has to process its corresponding subqueries with expected execution time $t_{x_{k}}$.

- Finally, the site formulates the final result of a global query from partial results received from other nodes or it can hold its own partial result waiting for other results. The expected execution time of this process is $t_{z}$.

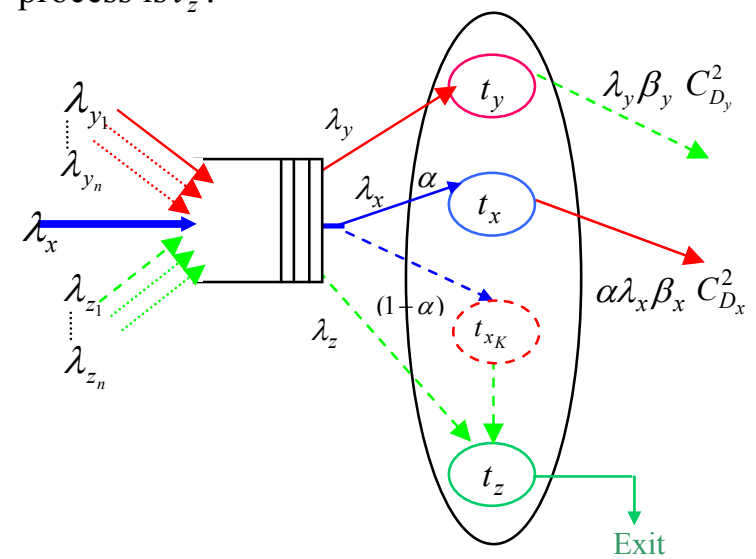

Figure 2: The Execution Queue

Calculations of the execution queue at node $k$ can be represented as follows:

- Total arrival rate of class $y$ is given by:

$$
\lambda_{y}=\sum_{i=1}^{n} \lambda_{y_{i}} \quad i \neq k
$$

- Total arrival rate of class $z$ is given by:

$$
\lambda_{z}=\sum_{i=1}^{n} \lambda_{z_{i}} \quad i \neq k
$$

Where node $k$ can receive subqueries and responses to subqueries from all the database nodes except itself.
- The arrival rate of class $x$ is $\lambda_{x}$ as the node can receive only one global query from a user at a time.

- The overall flow into the execution queue is given by: $\lambda_{S}=\lambda_{y}+\lambda_{x}+\lambda_{z}$

- The average execution time of processing subqueries received from other nodes is given by:

$$
\overline{t_{y}}=\frac{\lambda_{y}}{\lambda_{S}} t_{y}
$$

- The average execution time of processing global queries received from the user is given by:

$$
\overline{t_{x}}=\alpha \frac{\lambda_{x}}{\lambda_{S}} t_{x}
$$

- The average execution time of processing part of the global query locally if site $k$ is involved in it is given by:

$$
\overline{t_{x_{k}}}=(1-\alpha) \frac{\lambda_{x}}{\lambda_{S}} t_{x_{k}}
$$

- Average execution time of formalizing the final results or holding partial results received from other nodes is given by:

$$
\overline{t_{z}}=\frac{(1-\alpha) \lambda_{x}+\lambda_{z}}{\lambda_{S}} t_{z}
$$

- Total average execution time is given by:

$$
t_{S}=\overline{t_{y}}+\overline{t_{x}}+\overline{t_{x_{k}}}+\overline{t_{z}}
$$

- Utilization of process $y$ is given by:

$$
\rho_{y}=\lambda_{y} \overline{t_{y}}
$$

- Utilization of the process $x$ is given by:

$$
\rho_{x}=\alpha \lambda_{x} \overline{t_{x}}
$$

- Utilization of the process $x_{k}$ is given by:

$$
\rho_{x_{k}}=(1-\alpha) \lambda_{x} \overline{t_{x_{k}}}
$$

- The utilization of process $z$ is given by:

$$
\rho_{z}=\left((1-\alpha) \lambda_{x}+\lambda_{z}\right) \overline{t_{y}}
$$

- The total utilization of the execution queue over all classes is given by:

$$
\rho_{S}=\rho_{y}+\rho_{x}+\rho_{x_{k}}+\rho_{z}
$$

- The service coefficient of variation for process $x$ is given by:

$$
C_{\tau_{x}}^{2}=\frac{\operatorname{var}\left(t_{x}\right)}{t_{x}^{2}}
$$

- The service coefficient of variation for process $x_{k}$ is given by:

$$
C_{\tau_{x k}}^{2}=\frac{\operatorname{var}\left(t_{x k}\right)}{t_{x k}^{2}}
$$

- The service coefficient of variation for process $y$ is given by: 


$$
C_{\tau_{y}}^{2}=\frac{\operatorname{var}\left(t_{y}\right)}{t_{y}^{2}}
$$

- The service coefficient of variation for process $z$ is given by:

$$
C_{\tau_{z}}^{2}=\frac{\operatorname{var}\left(t_{z}\right)}{t_{z}^{2}}
$$

- The overall service coefficient of variation of all classes is given by [18]:

$$
C_{\tau}^{2}=\frac{\sum_{i=x, y, z, x_{k}} p_{i}\left(1+C_{\tau_{i}}^{2}\right) t_{i}^{2}}{t_{S}^{2}}-1
$$

- As an M/G/1 queue, the total Latency of the execution queue is given by [18]:

$$
T_{S}=t_{S}\left(1+\frac{\rho_{S}}{1-\rho_{S}}\left(\frac{1+C_{\tau}^{2}}{2}\right)\right)
$$

- The interdeparture coefficient of variation for class $\mathrm{x}$ is given by [18]:

$$
C_{D_{x}}^{2}=1+\rho_{x}^{2}\left(C_{\tau_{x}}^{2}-1\right)
$$

- The interdeparture coefficient of variation for class $\mathrm{y}$ is given by:

$$
C_{D_{y}}^{2}=1+\rho_{y}^{2}\left(C_{\tau_{y}}^{2}-1\right)
$$

The interdeparture coefficient of variation of classes $\mathrm{x}$ and $y$ from the execution queue will be the interarrival coefficient of variation of the same classes to the communication queue.

\subsection{Communication Queue}

As depicted in Figure 3, two classes flow into the communication queue. Class $y$ that represents responses to subqueries received from other nodes. Class $x$ that represents subqueries sent to other nodes from node $k$ as fragments from the global query submitted to node $k$. Having multiple channels and fully connected network, the communication queue calculations can be done as follows:

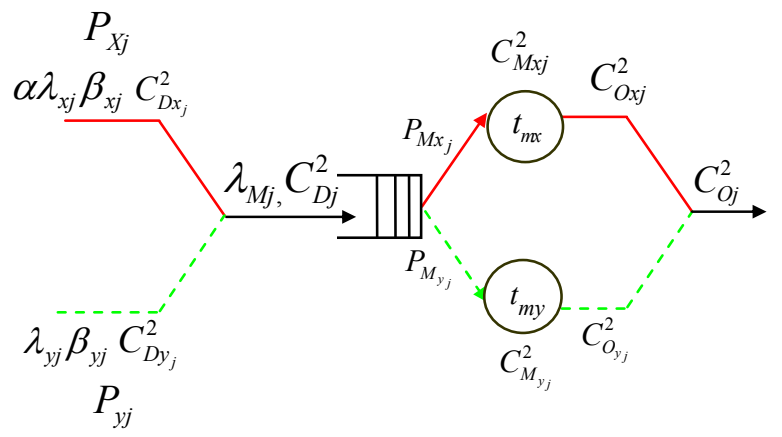

Figure 3: Communication Queue for Channel $\mathrm{j}$

- Flow into channel $\mathrm{j}$ over all classes is given by:

$$
\lambda_{M j}=\beta_{y} \lambda_{y} P_{y_{j}}+\alpha \beta_{x} \lambda_{x} P_{x_{j}}
$$

Where $\beta_{y} \lambda_{y}$ is the arrival rate from class $y$ to channel $j, P_{y_{j}}$ is the fraction of $\beta_{y} \lambda_{y}$ that is sent via channel this channel, $\beta_{x} \lambda_{x}$ is the arrival rate from class $x$ to channel $j$, and $P_{x_{j}}$ is the fraction of $\beta_{x} \lambda_{x}$ going into the channel.

- Using the split approximations [18], the interarrival coefficient of variation of the flows going into channel $j$ is given by:

$$
C_{D j}^{2}=C_{D y_{j}}^{2} P_{y_{j}}+C_{D x_{j}}^{2} P_{x_{j}}
$$

- Probability of getting a message from each class to channel $j$ is given by:

$P_{M y j}=\frac{\beta_{y} \lambda_{y} P_{y_{j}}}{\lambda_{M j}}, P_{M x j}=\frac{\alpha \beta_{x} \lambda_{x} P_{x_{j}}}{\lambda_{M j}}$

- The overall probability of a message going to cannel $j$ is given by:

$$
P_{M_{j}}=\frac{\lambda_{M_{j}}}{\lambda_{M}}=\frac{\lambda_{M_{j}}}{\sum_{j=1}^{L} \lambda_{M_{j}}}
$$

Where $\mathrm{L}$ is the number of channels.

- The expected communication service time for each class in channel $j$

$$
t_{m y j}=\frac{m_{y}}{R_{j}} \quad t_{m x j}=\frac{m_{X}}{R_{j}}
$$

Where $m$ is the message size and $R$ is the communication channel capacity.

- Average communication service time for channel $j$ over all classes is given by:

$$
t_{M j}=P_{M y j} t_{m y j}+P_{M x j} t_{m x j}
$$

- The utilization of channel $j$ is given by:

$$
\rho_{M j}=\lambda_{M j} t_{M j}
$$

- The average communication service coefficient of variation for channel $\mathrm{j}$ over all classes is [18]:

$$
C_{M j}^{2} \cong \frac{\left[P_{M y j}\left(1+C_{M y j}^{2}\right) t_{m y j}^{2}\right]+\left[P_{M x j}\left(1+C_{M x j}^{2}\right) t_{m x j}^{2}\right]}{t_{M j}^{2}}-1
$$

- As a $\mathrm{G} / \mathrm{G} / 1$ queue, the response time for channel $j$ is given by:

$$
T_{M j}=t_{M j}\left[1+\frac{\rho_{M j}\left(C_{D j}^{2}+C_{M j}^{2}\right)}{2\left(1-\rho_{M j}\right)}\right]
$$

- The overall response time for the communication queue over all channels is calculated as the weighted average of service time for each channel, and is given by: 


$$
T_{M}=\sum_{j=1}^{L} P_{M j} T_{M j}
$$

- The overall response time for a node is given by:

$$
T=T_{S}+T_{M}
$$

We calculated the interdeparture coefficient of variation per channel and then over the entire communication queue as they considered being the input to the next stage in the network.

- The inter-departure coefficient of variation for channel $j$ is given by which will be the input to the next stage [18]:

$C_{O j}^{2}=\rho_{M j}^{2} C_{M j}^{2}+\left(1-\rho_{M j}\right)\left(C_{D j}^{2}+\rho_{M j}\right)$

- The overall departure coefficient of variation for the communication queue can be estimated as [18]:

$$
C_{O}^{2}=\sum_{j=1}^{L} P_{M j} \cdot C_{O j}^{2}
$$

In these calculations we assumed a communication co-processor is available. In this case, communications has no impact upon processor availability (to the execution task). We also assumed that there is no effect from the interrupts and the available processing power is unity (e.g., $P=1.0$ ). If, however, the communication is supported by the execution queue (no communications processor available), we assume that the communications task is given highest priority. In this case, we have to incorporate the processing power effect within the behavior of the service activities.

\section{Efficiency of Multi-Level Caching in Distribued Systems}

In this section, we present the results of applying the multi-level caching architecture in a heterogeneous distributed system. These results are compared to the performance of single level caching and a regular query optimizer. We implement our model using the following system settings:

- The database is distributed among 20 sites that are connected via a fully connected network. The 20 sites are partitioned into three groups as shown in Table 1.

- The cache of each site is divided gradually into 7 linear levels. Each level is characterized by its search time and capacity.

\begin{tabular}{cccc}
\hline & No. of Nodes & $\begin{array}{c}\text { CPU } \\
\text { Rate }\end{array}$ & $\begin{array}{c}\text { Clock Cycle } \\
\text { Time }\end{array}$ \\
\hline Group One & 10 nodes & $2 \mathrm{Ghz}$ & $0.00005 \mathrm{~ns}$ \\
Group Two & 5 nodes & $2.5 \mathrm{GHz}$ & $0.00004 \mathrm{~ns}$ \\
Group Three & 5 nodes & $3 \mathrm{GHz}$ & $0.00003 \mathrm{~ns}$ \\
\hline
\end{tabular}

Table 1: Nodes Properties
- Assuming that we are using the LRU replacement policy, Table 2 gives the hit ratio for each level which is directly proportional to the level size [18].

- The arrival rate $\lambda$ and the probability of processing the global query at other sites $\alpha$ of each node are randomly generated.

- The probability of sending part of the global query from node $k$ to be processed at other sites is also generated randomly.

\begin{tabular}{cccc}
\hline Levels & Size & Hit Ratio & Latency \\
\hline L1 & $16 \mathrm{MB}$ & 0.014 & 1 Cycle \\
L2 & $32 \mathrm{MB}$ & 0.063 & 2 Cycles \\
L3 & $64 \mathrm{MB}$ & 0.114 & 3 Cycles \\
L4 & $128 \mathrm{MB}$ & 0,199 & 4 Cycles \\
L5 & $256 \mathrm{MB}$ & 0.322 & 5 Cycles \\
L6 & $512 \mathrm{MB}$ & 0.477 & 6 Cycles \\
L7 & $1 \mathrm{~GB}$ & 0.648 & 7 Cycles \\
\hline
\end{tabular}

Table 2: Cache Properties

Figures 4 and 5 demonstrate the efficiency of the proposed technique using the above system settings when increasing the number of caching levels from 0 , the regular Optimizer, to 8 caching levels varying the query size. The average query response time significantly decreases as the number of caching levels increases for different query sizes. It is clear that increasing the number of cache levels significantly improves the optimization time no matter how many numbers of query fragments we assume. For example, using 6 cache levels will reduce the optimization time $80 \%$ of the regular optimizer for any number of query fragments.

It can be noticed that the improvements in the optimization time when using multi-level caching starts to approach $90 \%$ saving after the seventh and the eighth levels.

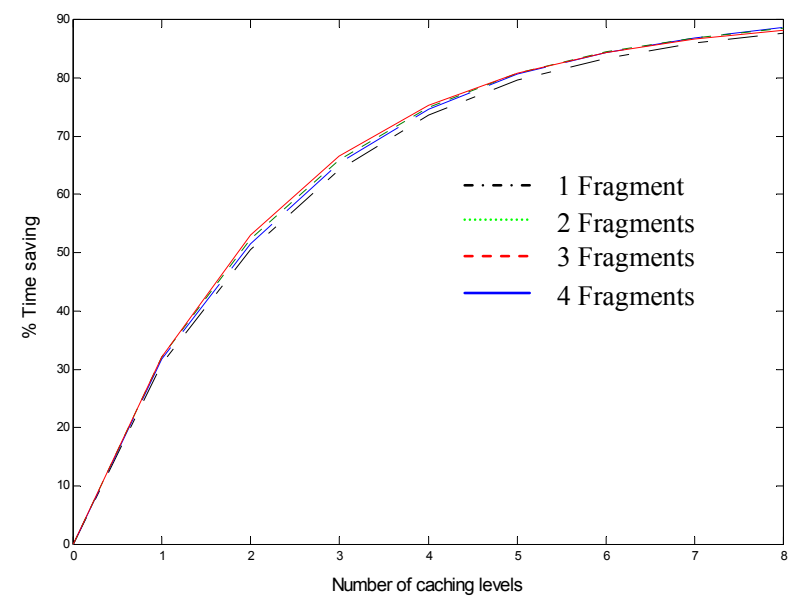

Figure 4: Percentage of time saving with respect to regular optimizer proportional to query size when using multi-level caching in a 20 -nodes heterogeneous network 


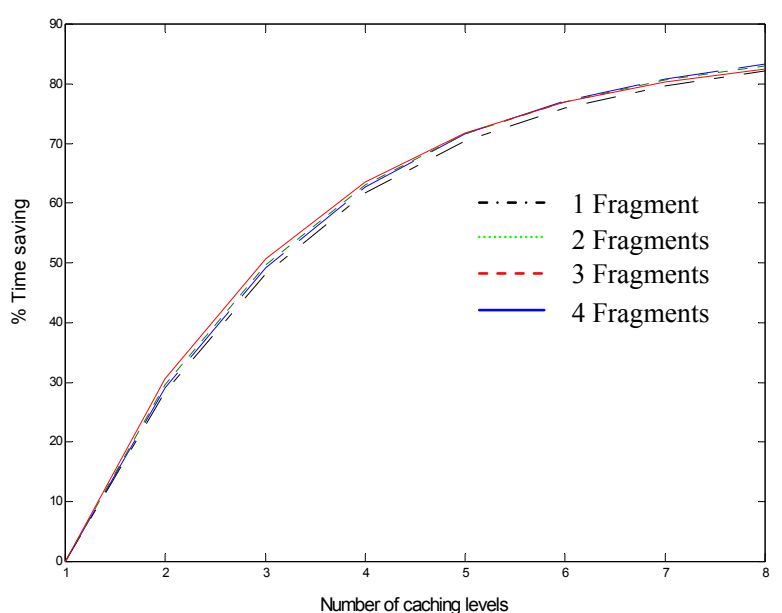

Figure 5: Multi-level caching versus single level caching varying the query size in a 20 -nodes heterogeneous network

\section{Conclusion}

In this paper, we present an analytical model to evaluate the performance of a new distributed query caching technique that is distinguished by caching query plans and results in a multi-level caching architecture [1]. Unlike other distributed database analytical models, we don't make the homogeneity assumption. Our model can be applied to different distributed database architectures. Our results show that caching query execution plans in a multi-level caching architecture significantly improves the query optimization time comparable to regular distributed query optimizers and single level caching.

\section{References}

[1] 1D.S.Elzanfally, A.S.Eldean, and R.Ammar. Multilevel Caching To Speedup Query Processing In Distributed Databases. in ISSPIT. 2003.

[2] D.S.Elzanfally, R.Ammar, and A.S.Eldin. Modeling and Analysis of a Multi-level Caching Database Systems. in The 46th IEEE International Midwest Symposium in Circuits \& Systems. 2003.

[3] S.Dar, M.J.Franklin, and B.T.Jonsson. Semantic Data Caching and Replacement. in 22nd VLDB. 1996. India.

[4] Q.Ren, M.H.Dunham, and V.Kumar, Semantic Caching and Query Processing. IEEE Trans. Knowledge and Data Eng, 2003. 15(1): p. 192210.
[5] F.N.Afrati, C.Li, and J.D.Ullman. Generating Efficient Plans for Queries Using Views. in Proc. ACM SIGMOD. 2001.

[6] D.Kossmann, M.Franklin, and G.Drasch. Cache Investment: Integrating query optimization and dynamic data placement. in ACM Trans. on Database Systems. 2000.

[7] P.Mitra. An Algorithm for Answering queries efficiency using views. in Proc. In The 12th Australasian Database Conf. (ADC'01. 2001.

[8] M.Nicola and M.Jarke, Performance Modeling of Distributed and Replicated Databases. IEEE Trans. Knowledge and Data Eng, 2000. 12(4): p. 645-672.

[9] E.G.Coffmann, E.Gelenbe, and BPlateau, Optimization of the number of copies in a distributed system. IEEE Trans. Knowledge and Data Eng, 1981. 7: p. 78-84.

[10] F.Bcelli and E.G.Coffmann, $A$ database replication analysis using an $M / M / m$ queue with service interruptions. Performance evaluation review, 1983. 11(4): p. 102-107.

[11] L.Kleinrock, Queueing systems, Volume I: Theory, ed. J.W. Sons. 1975.

[12] B.Ciciani, D.M.Dias, and P.S.Yu, Analysis of replication in distributed database systems. IEEE Trans. Knowledge and Data Eng, 1990. 2(2): p. 247-261.

[13] B.Ciciani, D.M.Dias, and P.S.Yu, Analysis of Concurrency-Coerency Control Protocols for Distributed Transaction Processing Systems with Regional Locality. IEEE Trans. Knowledge and Data Eng, 1992. 18(10): p. 899-914.

[14] J.M.Dermott and R.Mukkamala, Performance Analysis of Transaction Management Algorithms for the SINTRA Replicated ArchitectureDatabase Systems. IFIP Transzctions (Computer Science and Technology), 1994. A-47: p. 215-234.

[15] S.Banerjee, V.O.K.Li, and C.Wang, Performance analysis of the send-on-demand : A distributed database concurrency control protocol for high -speed networks. Computer Communications, 1994. 17(3): p. 189-204.

[16] S.Y.Hwang, K.S.Lee, and Y.H.Chin. Data Replication in a Distributed System: A Performance Study. in 7th Int. Conf. on Data and Expert Systems Applications. 1996.

[17] H.Sholl and I.Antonios, Two-Moment Hierarchical Queuing Model For the Task Level of the Hierarchal Performance Modeling System. 2000, Univ. of Connecticut, BRC/CSE-TR-00-1.

[18] Y.Zhou and J.F.Philbin. Eviction Based Cache Placement for Storage Caches. in Proceeding of the 2003 USENIX Annual Technical Conf. 2003. 